\title{
Signal superimpose system of micro-scale thermal imaging applied to un-cooled infrared cameras
}

\author{
by J. Morikawa*, E. Hayakawa** and T. Hashimoto** \\ * Tokyo Institute of Technology, Meguro-ku, Tokyo 152-8550, Japan, morikawa.j.aa@m.titech.ac.jp \\ **ai-Phase, Co., Ltd., Shinagawa-ku, Tokyo 141-0021, Japan
}

\begin{abstract}
Signal superimpose technique utilized in a micro-scale thermal imaging has been developed recently [1]. In this study the applicability of this technique to un-cooled micro-bolometer IR detector arrays is precisely examined. The method of pixel-by-pixel temperature calibration with superimposed temperature signal and a pseudo-acceleration of the sampling frame rate with a synchronized signal are applied to the precise measurement of phase change phenomena and the determination of the heat transport properties of the organic and polymeric materials.
\end{abstract}

\section{Introduction}

A recent development of thermal design on heat-conductive and thermal insulated materials requires a practical and precise determination method of heat transport properties. In many cases the inhomogeneous structures are designed in order to obtain the effective thermal conductivity and thermal diffusivity, in which the composition and the molecular orientation are highly controlled. From this perspective, a quantitative non-contact thermal imaging in microscale is promising to visualize the inhomogeneity of the thermal properties of the advanced materials.

A direct signal superimpose system has been developed and applied to a real time pixel-by-pixel temperature calibration and emissivity correction [1-14]. This technique is efficient when it is applied to observe the thermal phenomena during $1^{\text {st }}$ phase transition, as the temperature dependence of emissivity changes on phase transitions.

By utilizing this technique with the photon-type IR detector arrays, the visual thermal analysis, such like a timedependent two-dimensional differential scanning calorimetry (2D-DSC), has been realized. Furthermore, the Fourier analysis of thermal image by an input of the laser modulated spot heating visualizes the phase image of the inhomogeneous materials. The in-plane phase shift is correlated with the thermal diffusivity distribution in the plane surface of the sample.

On the basis of these techniques, the compact and portable-size equipment of a signal superimpose system with un-cooled micro-bolometer IR camera is designed, in which a pixel-by-pixel temperature calibration is applied and the spatial resolution of micro-bolometer is improved as $\sim 11 \mu \mathrm{m}$ with the optics optimized for the micro-scale thermography. In addition, in order to improve the time-scale resolutions for the periodic thermal event, an algorithm for "pseudo-acceleration" is applied to the un-cooled micro-bolometer system.

\section{Approaches}

Infrared thermography is typically applied by two approaches, passive and active methods. We aim at developing an analytical instrument of micro-scale Infrared thermography not only as an extension of these two approaches to micro-scale dimension, but also from the viewpoint of visual thermal analysis* in materials' industry. (* "Thermal analysis" is categorized as the method measuring the phase transition temperature, exothermic/endothermic enthalpy, and heat capacity under a temperature scan.) Typically, the passive approach investigates materials at some temperatures often higher than ambient temperature, while the active approach gives an external (thermal) stimulus in order to induce the thermal contrasts. As the third approach, in the case of visual thermal analysis, the stimulus is input by the temperature scan in the framework of linear response theory and the Duhamel's law of frequency-time transformation.

The concept of instrumentation of the analytical instrument for quantitative microscale thermography is aimed at two directions; active thermography, and visual thermal analysis. This is a common concept that can be applied to both a photon type and a thermal type IR detector arrays. In this study, originally developed two algorithms are examined, 1 Pixel by pixel emissivity calibration, and 2 Pseudo acceleration. From the viewpoint of general use in industry, the instrumentation with thermal IR detector arrays is available to realize the portable size analytical instrument.

The approach based on this concept is shown in the flowchart in Fig. 1. 


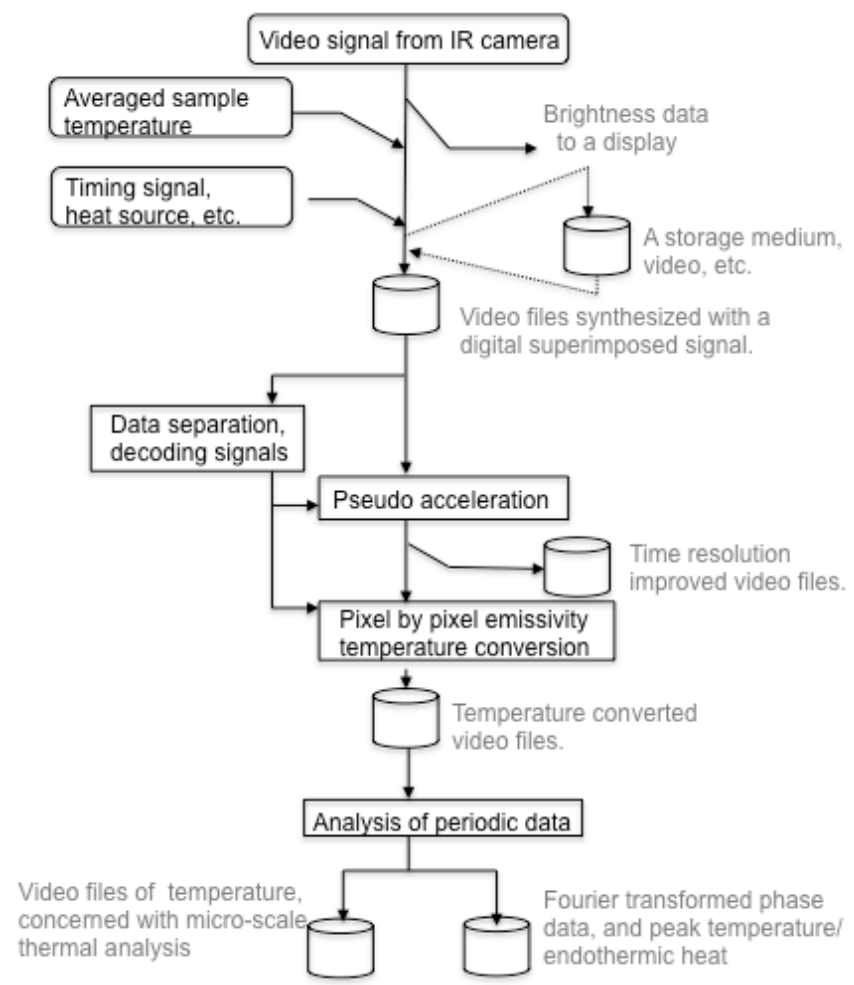

Fig. 1. Approaches of visual thermal analysis and pseudo acceleration with microscale thermography using thermal detector arrays.

\section{System of signal superimpose}

The measuring instrument is composed of

A: Signal (NTSC, LVDS) capturing part,

$B$ : Superimposer of video signal,

C: Timing trigger generator,

D: Synchronous IR camera (VOx FPA 480 x 640 pixel numbers, cf. InSb FPA 640 x 512), controller

E: High-precision / high-speed temperature

$\mathrm{F}$ : Acutuator and laser drive,

G: Stage scanners, for VOx),

$\mathrm{H}$ : Optics (microscopic lens: $\times 10$ for $\operatorname{lnSb}$, x 2.5

I: a cassette-type chamber as a sample-holder, Figure 2.

as depicted in the schematic view of a setup in

The laser drive $\mathrm{F}$ (in Fig.2) for generating a modulated spot heating with $630 \mathrm{~nm}$ diode laser and the xy positioning actuator are triggered with a timing signal synchronous to the NTSC image capturing (Fig. 3). The video signal synthesizer enables to record a direct signal

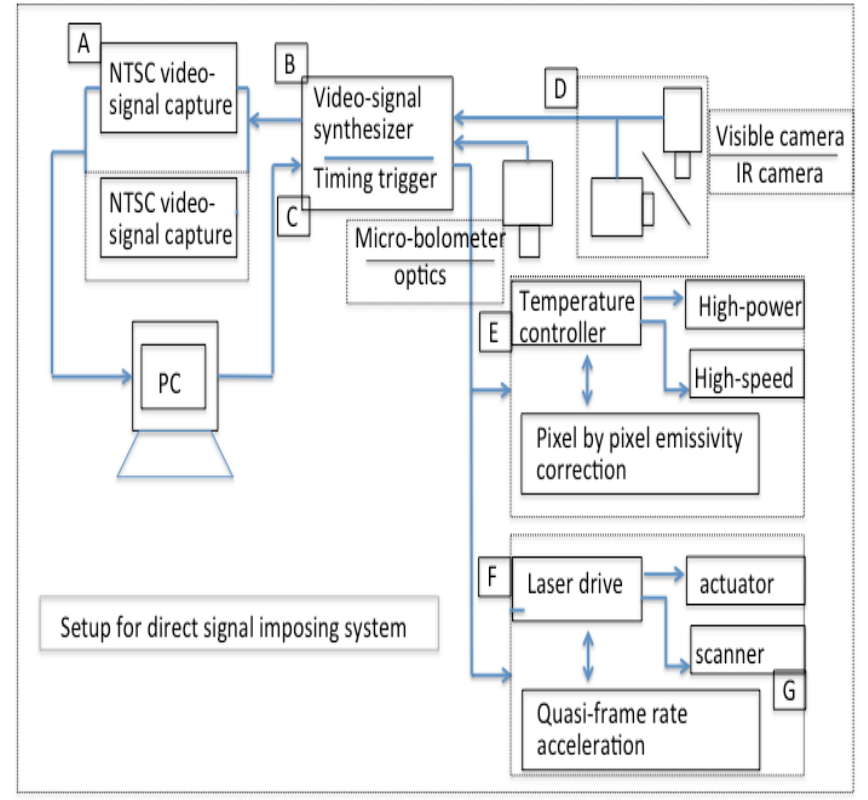

Fig. 2. Schematic view of a setup. monitoring of temperature or positioning data, simultaneously, that is measured with different sensor systems. The imposed data are applied to the pixel emissivity corrections at one-time under heating/cooling temperature scan. The Peltier-type dual heater is set to operate a heating and cooling scan of the specimens. 
The high-precision / high-speed temperature controller $\mathrm{E}$ (in Fig. 2) is designed as a one-type cassette-type chamber as depicted in Figure 3. The actuator/ laser drive $(F)$ and a stage scanner $(G)$ are combined as another cassette-type chamber I as a sample-holder. A dimension of the cassette chamber is $3 \mathrm{~cm} \times 3$ $\mathrm{cm} \times 2 \mathrm{~cm}$.

By exchanging the cassette chamber for the purpose of the measuring, different approaches of microscale thermography are realized in one portable instrument, one as a visual thermal analysis, and the other as a passive thermography with an input from the modulated diaode laser source. The portable-size design with un-cooled VOx FPA is $18 \mathrm{~cm} \times 21 \mathrm{~cm} \times 14 \mathrm{~cm}$ (Fig. 3).

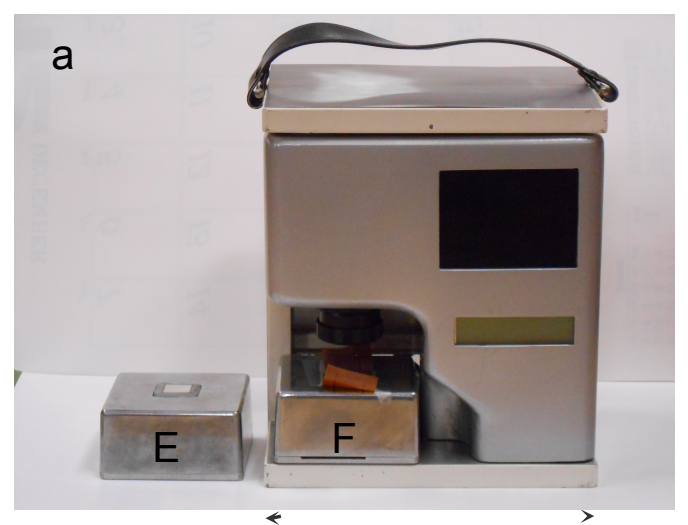

$18 \mathrm{~cm}$
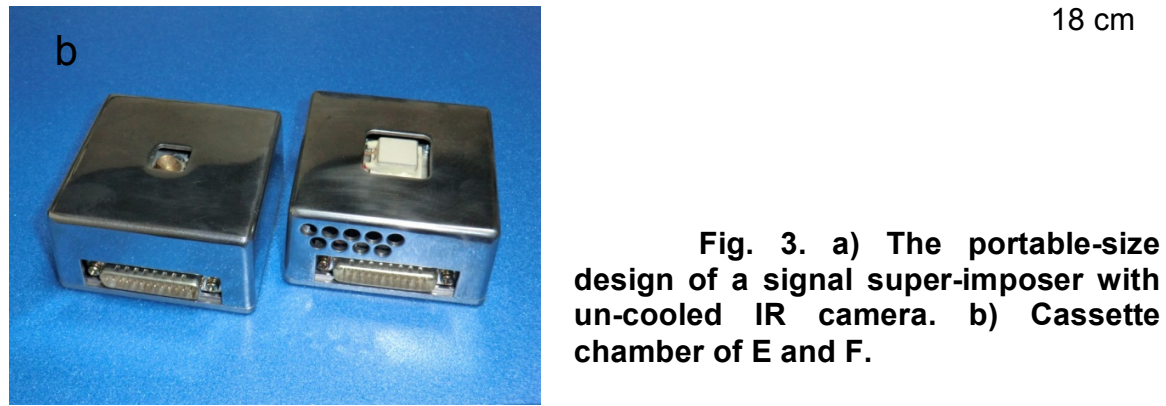

\subsection{Superimpose}

The direct signal superimpose system is composed of the video-signal synthesizer, the timing trigger, and NTSC video-capturing hardware connected to IR camera data processing system (Fig. 4a). The video signal synthesizer records a direct digital signal of temperature and positioning sensor. The encoded digital signal data embedded in each image (Fig. 4b)is decoded to read out on the analysis. The protocol to encode/decode data is originally defined. The mixed signals of IR detector arrays and the imposed data are applied to the pixel-by pixel emissivity corrections and the pseudo-acceleration of the periodical thermal phenomena.

\section{a. Signal synthesis}

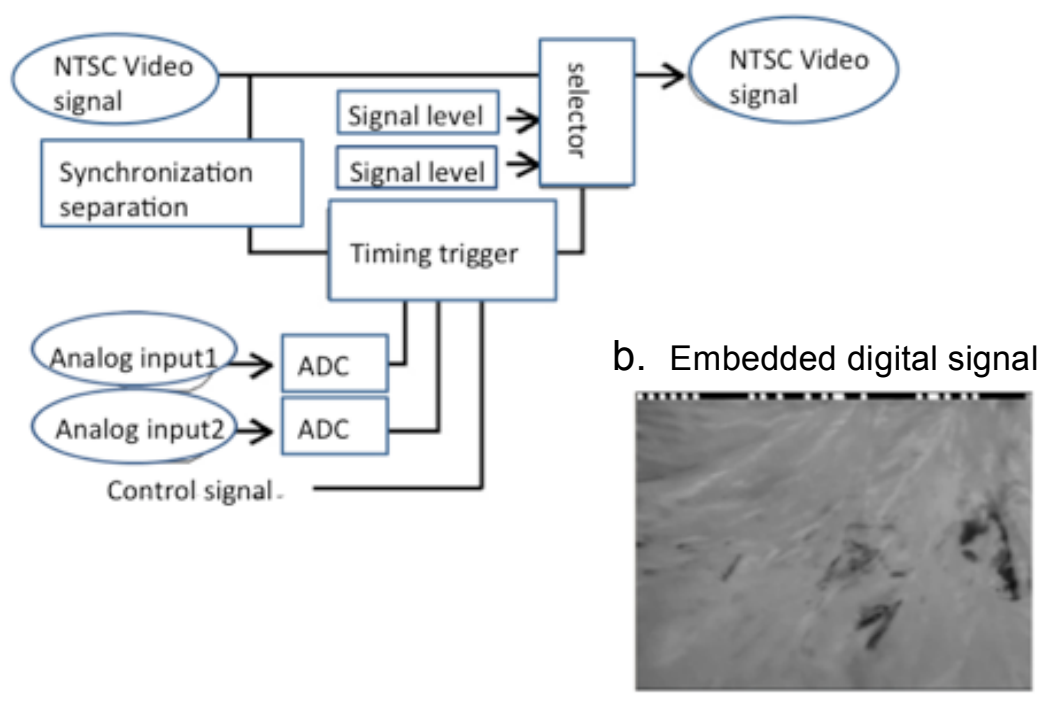

Fig. 4. Scheme of the signal synthesis.

\subsubsection{Video-signal Superimpose}


The brightness data is serial, synchronized to the data clock, with16 bit per1 pixel. The frequency of data clock is $70 \mathrm{MHz}$ or $200 \mathrm{MHz}$. The synchronized signal of VSYNC, and HSYNC of NTSC signal are included in Data sync signal.

The signal synthesizing function replaces the data in the imposed area from the camera input (data clock, data out, and data sync), into the imposed pixel data as a data output. The signal synthesizing process is schematically shown in Fig. 5.

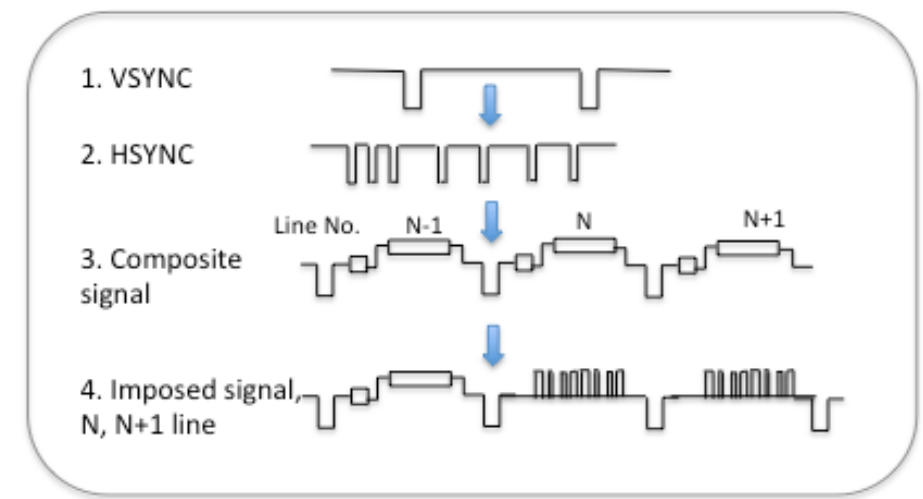

Fig. 5 Scheme of the video-signal synthesizer

\subsubsection{Pseudo-acceleration}

The algorithm for "pseudo-acceleration" is developed in order to improve the time-scale resolutions for the periodic thermal event such as a modulated spot heating. Generally, as the capturing timing of camera and the periodic heat source driving are not synchronized, there exists a variable time delay. However, if each image that is captured in each cycle is re-ordered according to the periodically normalized time over some cycles, the time resolution is apparently improved. It contributes to reduce the noise by integrating the multiple image data, keeping a time resolution. (Usually, the exposure time is increased in order to reduce the noise, which damages the time resolution.)
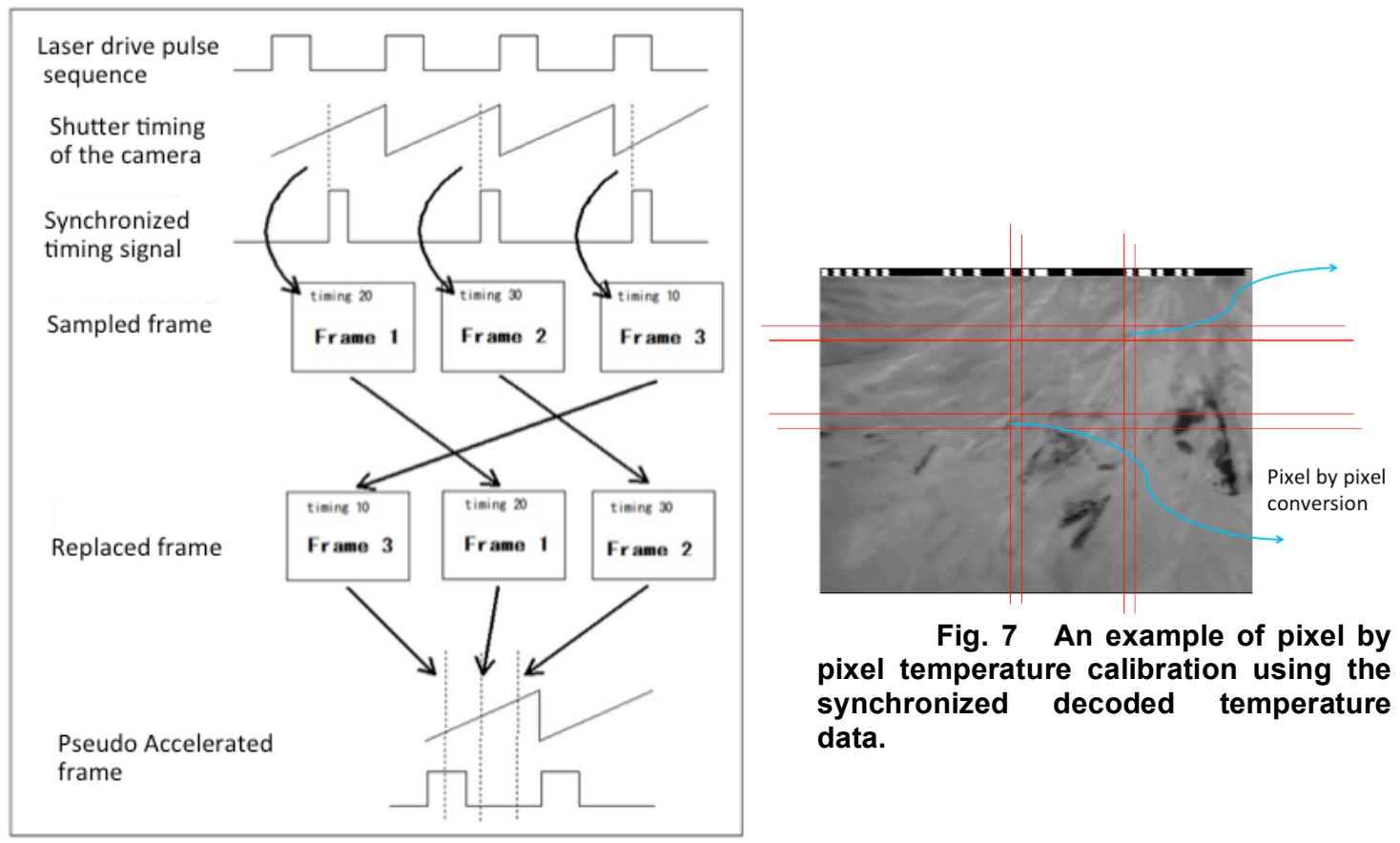

Fig. 7 An example of pixel by pixel temperature calibration using the synchronized decoded temperature data.

Fig. 6 Scheme of the principle of "pseudo-acceleration) of periodic thermal events.

\subsubsection{Pixel by pixel temperature calibration}


Pixel by pixel temperature calibration is carried out using the superimposed video-signal of temperature. By using the imposed signal, the measured intensity of each pixel is directly calibrated with a temperature dependent emissivity correction factor. This calibration technique is advantageous for the measurement under a temperature scan with phase change or a modulated temperature field. The direct superimpose technique enables the high-resolution of spatial and time directions.

\section{Results}

\subsection{Pseudo acceleration of a modulated heat spot input on the polymer film}

The effect of pseudo-acceleration on Fourier transformed image is examined in comparison with the raw data and the transformation. Figure 8 shows those images measured on the polyimide film based copper leads laminate for flexible printed wiring boards irradiated with a modulated spot laser heating at $0.87 \mathrm{~Hz}$. In the pseudo-accelerated images $(E \sim G)$, especially in the $2^{\text {nd }}$ harmonic phase image, the noise is reduced by the pseudo-acceleration compared with the image in $\mathrm{C}$ without the pseudo-acceleration. The results indicate a potential of the micro-scale thermal imaging with micro-bolometer as the quantitative analysis. The thermal interface between metal and polymer is also clearly visualized with this noise reducing method.
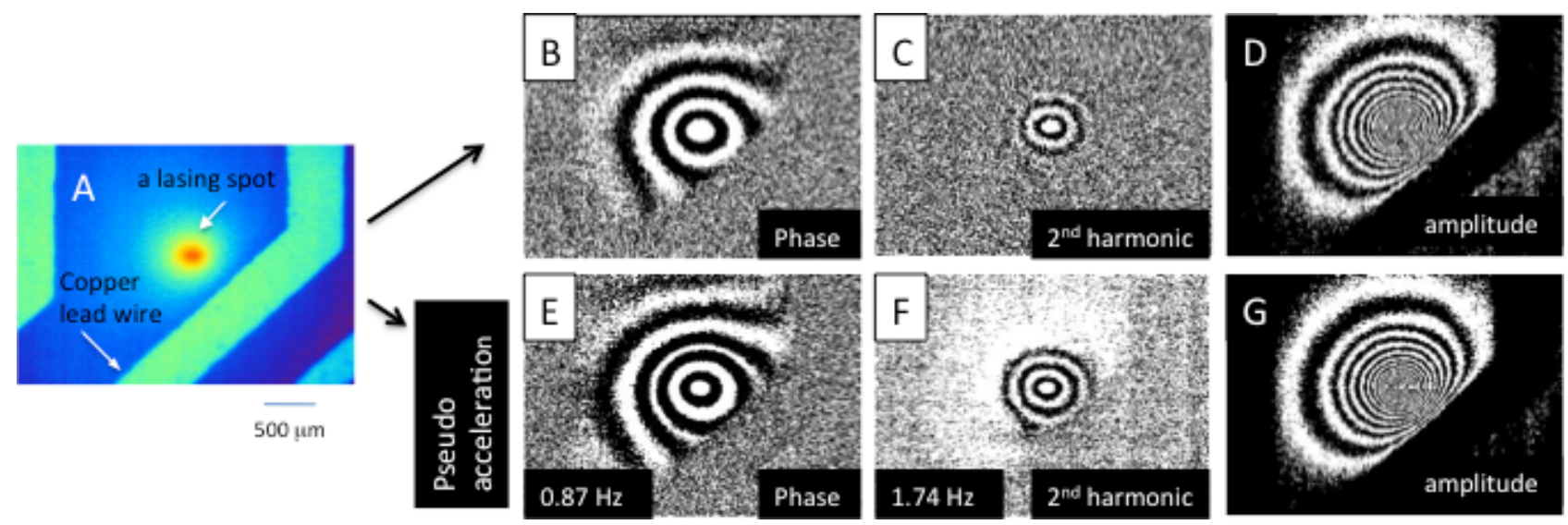

Fig. 8. Effect of pseudo acceleration on Fourier transformed phase and amplitude images of polyimide film based copper leads laminate for flexible printed wiring boards irradiated with a modulated spot laser heating at $0.87 \mathrm{~Hz}$. A: raw image, B D: Fourier transformed phase and amplitude images, E G: Pseudo accelerated image with Fourier transform. $C$ and $F$ are the $2^{\text {nd }}$ harmonic phase image. The contour line is depicted in the figures.

\subsection{Afterimage of the continuous scanning of a modulated heat spot}

By scanning a modulated heat spot in the $x-y$ direction on the surface of the specimen, using a micro-scale stage scanner equipped in the cassette chamber $F$, a movement afterimage of thermal wave is observed. The amplitude of input diode laser is modulated at a fixed frequency, and the scan is periodically controlled in one direction. As a consequence a double modulation of heat spot is input in the specimen. The phase lock-in calculation is executed in the chosen timeframe corresponding to a location.

The different pattern of phase and amplitude is obtained depending on the scan speed and the modulated frequency. One example is shown in Fig. 9, where the input frequency is $0.87 \mathrm{~Hz}$, the scan rate is $755 \mu \mathrm{m} / \mathrm{s}$ at a frequency $0.2 \mathrm{~Hz}$ in the observed micro-scale area. The scanning thermal image is shown in Fig. $9 \mathrm{~A} \sim \mathrm{C}$ in each cycle.

Fourier analysis in time direction gives the phase image. The results are shown in Fig. $9 \mathrm{D} \sim \mathrm{F}$ as a contour line plot. Because of the waveform of an input heat source the higher harmonics are obtained as observed in Fig. $9 \mathrm{E} \& \mathrm{~F}$. In Fig. 9D, a wedge shape contour line of phase is observed. When the wedge is a straight line, it means that the specimen is homogeneous around the heat spot. The angle of the wedge line and the horizontal line is about 45 deg, which suggests that the scan speed of the heat spot in X direction is close to the thermal diffusion rate in the y-axis direction is close.As a fact, the thermal diffusion length in in-plane surface of the specimen is close to the scan rate in x-direction. By judging the contour line and the angle to the horizontal line, the inhomogeneity and the thermal diffusion rate on the specimen film is examined. Pseudo acceleration technique is to be applied to the scan mode measurement in order to improve the sensitivity. 


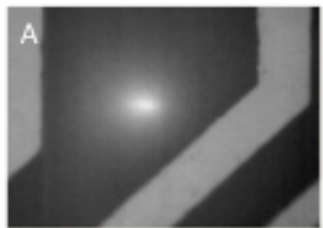

$\mathrm{t}=\mathrm{t} 1$

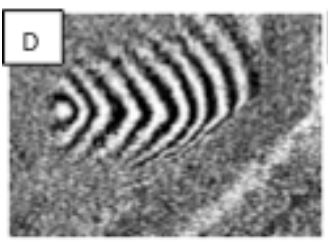

$1^{\text {st }}$ phase

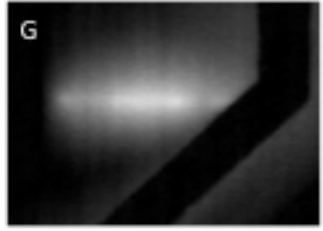

$1^{\text {st }} \mathrm{amp}$.

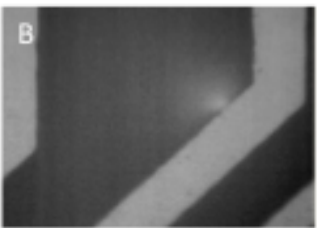

$\mathrm{t} 2=\mathrm{t} 1+1.3 \mathrm{~s}$

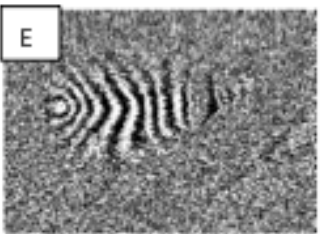

$2^{\text {nd }}$ phase

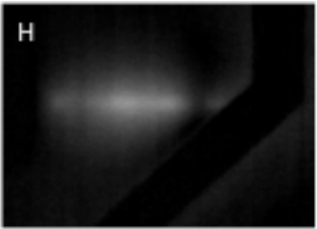

$2^{\text {nd }} a m p$

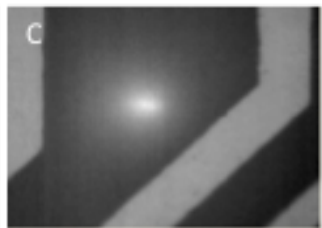

$\mathrm{t} 3=\mathrm{t} 2+2.6 \mathrm{~s}$

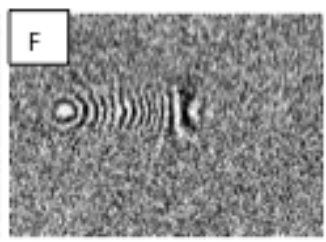

$3^{\text {rd }}$ phase

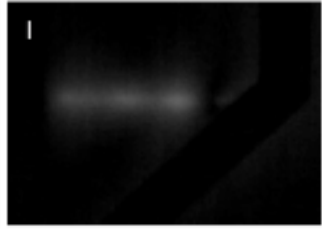

$3^{\text {rd }} \mathrm{amp}$
Fig. 9. Afterimage of thermal wave with an input of a modulated heat spot, scanning in $x$ direction at a constant rate on the surface of the polyimide film based copper leads laminate for flexible printed wiring boards. The modulated frequency is $0.87 \mathrm{~Hz}$. A speed of scan is $755 \mu \mathrm{m} / \mathrm{s}$. A C: raw image, D F: Fourier transformed phase image with a contour line, G I: Fourier transformed amp image. The scale bar is the same asdepicted in Fiq. 8.

\section{Conclusion}

The direct signal superimpose technique is developed and applied to a micro-scale thermal imaging system with un-cooled micro-bolometer arrays. The pseudo acceleration of the frame rate in the cyclic thermal event and a pixel by pixel temperature calibration in visual thermal analysis are successfully introduced. It will provide a practical and a precise imaging method for the advanced thermal characterization in materials industry.

\section{REFERENCES}

[1] Morikawa, J., Hayakawa, E., Hashimoto, T, " Two-dimensional Thermal Analysis of Organic and Polymeric Materials with cooled and uncooled infrared cameras". Advances in Optical Technologies 2012, 484650, 2012.

[2] A. Rogalski, J. Antoszewski, and L. Faraone, "Third-generation infrared photodetector arrays," J. Appl. Phys., vol. 105, pp. 091101-1-44, 2011.

[3] T. Hashimoto, J. Morikawa, " Two-dimensional thermal analysis on freezing of onion epidermal cell by highspeed infrared microscopic camera," Jpn. J. Appl. Phys., vol. 42, L706-L708, 2003.

[4] J. Morikawa, T. Hashimoto, E. Hayakawa, H. Uemura, "Two-dimensional thermal analysis for freezing of plant and animal cells by high-speed microscopic IR camera," SPIE vol. 5073, 148-153, 2003.

[5] J. Morikawa, T. Hshimoto, K. Yamamoto, J. Ando, "Tow-dimensional thermal analysis for freezing of endothelial cells by high-speed microscopic IR focal plane arrays," SPIE vol. 5697, pp. 282-290, 2005.

[6] C. Pradere, J. Morikawa, J. C. Batsale, T. Hashimoto, "Microscale thermography of freezing biological cells in view of cryopreservation," Quantitative Infra Red Thermography Journal vol. 6, pp.37- , 2009.

[7] J. Morikawa, T. Hshimoto, E. Hayakawa, T. Eto, " Two-dimensional thermal analysis of organic materials by micro-scale thermography", QIRT 2008 Conference, Krakov, Poland, 2-5 July, 2008, paper pp. 397 - 401.

[8] J. Morikawa, E. Hayakawa, K. Ikuo, T. Hashimoto, " Two-dimensional thermal analysis of organic molecular crystals and polymeric spherulites by microscale thermography", SPIE vol. 7661, pp.76610S-1-9, 2010.

[9] J. Morikawa, E. Hayakawa, T. Hashimoto, "Application of micro-scale thermography to the thermal analysis of polymeric and organic materials", SPIE vol. 8013, pp. 801319-1-6, 2011.

[10] J. Morikawa, E. Hayakawa, T. Hashimoto, R. Buividas, S. Juodkazis, " Thermal imaging of a heat transport in regions structured by femtosecond laser", Opt. Exp. 19, 20542-20550, 2011.

[11] J. Morikawa, T. Hashimoto, " Thermal imaging of micro-structured polymers with high-speed infrared camera", Proc. SPIE. 8204, Smart Nano-Micro Materials and Devices 82042R (December 21, 2011).

[12] J. Morikawa, E. Hayakawa, T. Hashimoto, " Microscale thermal analysis with cooled and uncooled infrared cameras ", Proc. SPIE. 8354, Thermosense: Thermal Infrared Applications XXXIV 835410 (May 18, 2012).

[13] J. Morikawa, " Micro-scale thermal imaging of advanced organic and polymeric materials", Proc. SPIE. 8511, Infrared Remote Sensing and Instrumentation XX 85110T (October 24, 2012).

[14] J. Morikawa, E. Hayakawa, T. Hashimoto, " Two-dimensional Thermal Analysis of Organic and Polymeric Materials with cooled and uncooled infrared cameras", Advances in Optical Technologies 2012, 484650 (2012). 